\title{
Radiotherapeutic Alternatives to Standard Management of Adenocarcinoma of the Endometrium
}

\author{
Willie A. Andersen, M.D., ${ }^{*, 1}$ William A. Peters III, M.D., $†$ Robert E. \\ FechNer, M.D. $\ddagger$ GeOrGe W. Morley, M.D. $\dagger$ AND \\ W. NoRMan ThORNTON, JR., M.D.* \\ Departments of *Obstetrics and Gynecology and $\ddagger$ Pathology, University of Virginia Medical \\ Center, P. O. Box 465, Charlottesville, Virginia 22908, and $\dagger$ Departments of Obstetrics and \\ Gynecology, University of Michigan School of Medicine, Ann Arbor, Michigan 48109
}

Received August 13, 1982

\begin{abstract}
Primary radiotherapy as an alternative management for adenocarcinoma of the endometrium was chosen for 117 patients treated at the University of Michigan Medical Center and University of Virginia Medical Center. Cases were selected for radiation because of contraindications to surgery $(52.2 \%)$ or by protocol for disease outside the endometrium $(47.8 \%)$. An overall 5 -year actuarial survival rate of $49.6 \%$ was attained for all stages, with 55\% 5-year survival for disease limited to the uterus or cervix. Stage and grade were the most significant risk factors. The addition of external-beam irradiation did not improve local failure rates or survival. Heyman's uterine packing technique was slightly more successful than uterine "line sources" in controlling local disease $(P=0.08)$. Treatmentrelated mortality $(0.8 \%)$ and morbidity $(6.8 \%)$ were minimal. Surgery, whenever possible, remains the "best standard therapy" but the radiotherapeutic alternative is of significant benefit for those deemed nonsurgical candidates.
\end{abstract}

\section{INTRODUCTION}

Forty years have passed since Professor Heyman presented the results of the radiotherapeutic treatment of adenocarcinoma of endometrium and demonstrated the radium packing technique to be, in essence, as efficacious as total hysterectomy in the successful management of this malignancy [1]. Much has changed in medicine since then. Treatment results with surgery are now universally accepted as superior to radiation therapy alone. There remains, however, a small group of patients with corpus cancer who even today have contraindications to conventional management (i.e., TAH/BSO) because of either significant medical complicating factors or unresectable lesions. Although gynecologic surgeons and gynecologic oncologists continue to need alternatives to abdominal hysterectomy in the treatment of these selected high-risk patients, relatively few large series that incorporate modern radiotherapy technology are available to assess primary radiation for endometrial cancer [2-4]. A retrospective clinical review is therefore reported to

\footnotetext{
${ }^{1}$ To whom requests for reprints should be addressed.
} 
(1) examine the selection of patients for therapy with radiation alone,

(2) evaluate the success of primary radiation therapy,

(3) assess the radiotherapeutic methods most successful in achieving local control,

(4) assess the influences of megavoltage external therapy that has developed since Heyman's initial report,

(5) assess the cost of therapy in terms of treatment morbidity and mortality.

\section{MATERIALS AND METHODS}

To maximize the study population, the clinical experience of the gynecologic oncology units of the University of Michigan and the University of Virginia were combined. From 1950 through 1979, 126 patients with endometrial adenocarcinoma were treated with radiation therapy alone at the two institutions. Histopathologic review excluded 9 patients who on reevaluation had either hyperplasia ( 5 cases) or metaplasia (4 cases). In the remaining 117 cases, 58 separate variables were abstracted and subsequently computer analyzed.

Radiation alone was chosen based on general clinical characteristics that predicted poor tolerance of standard therapy, i.e., hysterectomy, although at one time all Stage II corpus cancers were selected for radiation therapy at both institutions. In addition, patients with Stage III and Stage IV disease were treated with radiation for control of pelvic disease. Six patients had prior pelvic radiation therapy ( 4 for malignancy, 2 for benign conditions) and were retreated with radiation after they developed a uterine adenocarcinoma.

The pretreatment radiotherapy prescriptions, although quite varied, were all designed to at least effect local control. The treatment schema fell into three broad categories. Intracavitary radium application alone utilizing either Heyman's capsules or Campbell's capsules, or uterine tubes and vaginal vault sources (rarely whole-length vaginal cylinders) were prescribed to deliver 6000-7000 rad to point $A$ or $5000-6000 \mathrm{mg} / \mathrm{hr}$ in one or two packings. If a uterine tube was used, loading of the sources was such that a more cylindrical dose distribution was obtained, in contrast to standard loading for cervical lesions [5]. A second approach was to use intracavitary radium application to deliver 3000-4000 rads to point $\mathrm{A}$ or $4000-5000 \mathrm{mg} / \mathrm{hr}$ coupled with external-beam therapy to the whole pelvis, 4000-5000 rad total midline dose over 4 to 5 weeks. Finally some patients were treated with whole-pelvic irradiation only, usually to a dose of 5000-6000 rad total midline dose over 5 to 10 weeks.

\section{RESULTS}

\section{General Observations}

Of the 117 patients treated with radiation therapy alone, only about one-half were Stage I (Table 1), about $35 \%$ were Stage II, and about $12 \%$ had extrauterine disease (Stages III and IV). The corresponding actuarial survival probability (Berkson and Gage method) by stage, although not surprising, is dramatically significant at $P$ greater than 0.0001 using the Cox's life table regression model, and represented a powerful confounding influence when analyzing other variables affecting outcome. The next most powerful determinant of survival probability 
TABLE 1

Stage Distribution and Actuarial Survival Probability for 117 Patients Treated with RADIOTHERAPY ONLY

\begin{tabular}{lccc}
\hline Stage & Patients & $\begin{array}{c}\text { 5-Year survival } \\
\text { probability } \\
\%\end{array}$ \\
\hline IA & Number & $\%$ & 61.8 \\
IB & 35 & 29.9 & 67.9 \\
II & 26 & 22.2 & 42.3 \\
III & 42 & 35.9 & 18.0 \\
IV & 10 & 8.5 & 0.0 \\
Total & 4 & 3.4 & 49.6 \\
\hline
\end{tabular}

in this series was histologic grade. It was more significant than histologic type. Grading of the tumors incorporated characteristics of cytoplasmic and nuclear size, variability, and atypicality, and not pattern of growth alone [6]. Less than one-half of the patients had well-differentiated lesions while one-fourth of the cases were poorly differentiated (Table 2). Only $32 \%$ of the patients with G-III lesions survived their disease, compared to $57 \%$ for patients with more favorable differentiation $(P=0.05)$. Table 2 collates survival by grade in patients with disease limited to the uterus, i.e., Stages I and II.

The histologic types encountered (Table 3) reflect a broad spectrum. Of interest, there was a history of prior pelvic RT noted in 2 of 12 cases of clear cell adenocarcinoma, in 1 of 13 cases of papillary carcinoma, but in only 3 of 75 cases of the "endometrial"-type adenocarcinoma. Predicted 5-year survival for the "endometrial" adenocarcinoma was $52.4 \%$, for adenosquamous carcinoma, $44.7 \%$, and for clear cell adenocarcinoma, 37\%. Papillary carcinoma had an unexpected survival prediction of $70.08 \%$ but $54 \%$ of these cases were Stage I.

Pretreatment uterine size (estimated in equivalent gestational age/size in weeks) ranged from normal to 20 weeks for the group, with a median of 8 weeks. Uterine sounding values ranged from 4 to $16 \mathrm{~cm}$ (median $8 \mathrm{~cm}$ ). Corpus dimensions by

TABLE 2

Histologic Type and Cytologic Grade of the 117 Patients Treated with Radiotherapy Alone ${ }^{a}$

\begin{tabular}{lrrrr}
\hline & \multicolumn{3}{c}{ Cytologic grade } & Total \\
\cline { 2 - 5 } Histologic type & G1 & G2 & G3 & 70 \\
\hline Endometrial & 34 & 23 & 13 & 11 \\
Clear cell & 7 & 1 & 3 & 13 \\
Papillary & 7 & 5 & 1 & 12 \\
Adenosquamous & 0 & 1 & 11 & 2 \\
Mucinous & 0 & 1 & 1 & 1 \\
Undifferentiated & 0 & 0 & 30 & 109 \\
Total & 48 & 31 & & 1 \\
\hline
\end{tabular}

${ }^{a}$ Eight patients, data not known. 
TABLE 3

Patients with Disease Limited to the Uterus: Survival by Stage and Grade

\begin{tabular}{lc}
\hline & $\begin{array}{c}\text { 5-Year survival probability } \\
(\%)\end{array}$ \\
\hline Stage I & \\
Grade I & 67.1 \\
Grade II & 75.9 \\
Grade III & 50.6 \\
All grades & 64.5 \\
Stage II & \\
Grade I & 57.3 \\
Grade II & 55.7 \\
Grade III & 23.0 \\
All grades & 42.3 \\
\hline
\end{tabular}

sounding seemed retrospectively to have influenced choice of therapy; i.e., smaller uteri were generally chosen for intracavitary treatment alone, and therapy for larger uteri more often incorporated internal and external therapy or external therapy alone (correlation $P=0.05$ ). Uterine size estimates, however, showed no correlation with choice of radiation therapy technique. In addition the type of therapy chosen appeared to be independent of the histologic grade of the tumor, and the histologic type of tumor. The stage of endometrial cancer prior to treatment did show a significant influence on choice of therapy $(P=0.05)$ with the more advanced stages relying primarily on external beam alone.

\section{Clinical Characteristics with "Medical Contraindications" to Surgery}

The 61 cases of Stage I endometrial carcinoma in this study were all selected for radiation therapy purely on the basis of predicted unacceptable morbidity and mortality from surgery. The clinical profile of these patients (Table 4) indeed reflects a medically high-risk group. Over one-half of these patients were above the seventh decade of life and because of their general health problems over one-half were confined to bed for the greater part of each day. The typical patient had at least three of the medical diagnoses tabulated.

Twelve of the sixty-one patients had a prior history of cancer (four presumed cervix, three breast, two lymphoma/leukemia, one each colon, melanoma, sinus) and three additional patients had concominant second primaries (two breast, one thyroid). One patient developed gastric carcinoma after successful treatment of her endometrial cancer. Fifteen of these sixteen patients died of malignancy, but only five died of progressive uterine disease.

Although 22 of the patients with medical contraindications to surgery succumbed to endometrial malignancy, $31(51 \%)$ died as a result of other conditions, free of cancer. Mean survival of those dying of malignancy was 49.3 months, while mean survival of those dying of other nonmalignant conditions cancer free was 71.2 months $(P=0.08)$. 
TABLE 4

Clinical Profile of the 61 Patients Selected for Primary Radiotherapy because of Medical Contraindications ${ }^{a}$

\begin{tabular}{lcr}
\hline & \multicolumn{2}{c}{ Patients } \\
\cline { 2 - 3 } Medical condition $^{b}$ & Number & $\%$ \\
\hline High blood pressure & 44 & 72 \\
Obesity & 29 & 48 \\
Other $^{c}$ & 28 & 46 \\
Diabetes mellitus & 27 & 44 \\
Coronary disease & 22 & 36 \\
Pulmonary disease & 6 & 10 \\
Cerebral vasc disease & 5 & 8 \\
Dementia & 3 & 5 \\
\hline
\end{tabular}

${ }^{a}$ Median age was 71.7 years (range $41-87$ ) and median performance status was 3 (GOG scale).

${ }^{b}$ Many patients had more than one condition.

c Other includes patients with one or more of the following: 15 with prior malignancy, 10 with arthritis, 10 with congestive heart failure, 3 with deep vein thrombophlebitis, and 3 with pulmonary embolus.

\section{Analysis of Treatment Success and Failure}

In order to analyze treatment factors that influenced local control and survival, patients with disease clinically confined to the uterus or cervix (Stages I and II) were grouped together $(N=103)$. Patients with extrauterine disease (Stages III and IV) in this series had a predicted 5-year survival of only $13 \%$ contrasted to $55 \%$ for those with disease limited to the uterus and/or cervix $(P>0.005)$. Although $38.8 \%$ of Stage I and II cases died of disease, 33\% died of other causes, tumor free. Table 5 illustrates the pattern of treatment failure in Stage I and II. Disease recurrences in the uterus, cervix, upper vagina, or pelvic side wall (pelvic recurrence) strongly correlated with the likelihood of extrapelvic (groin, intraabdominal, liver or lung) metastatic disease as well $(P>0.01)$. Of the patients

TABLE 5

Pattern of Recurrences in 103 Patients with Stage I and II Adenocakcinoma of the Corpus TREATED WITH RADIOTHERAPY ALONE ${ }^{a}$

\begin{tabular}{lccc}
\hline & & Extrapelvic recurrence & \\
\cline { 2 - 4 } Pelvic recurrence & No & Yes & Total \\
\hline No & 54 & 11 & 65 \\
Yes & 12 & 14 & 26 \\
Total & 66 & 25 & 91 \\
\hline
\end{tabular}

${ }^{a}$ Excluded are 9 cases of recurrence but site not known and 3 cases of persistent disease. 
with recurrent disease, the median recurrence-free interval was 11.1 months after initial treatment.

The local failure (defined as locally persistent or locally recurrent tumor within the pelvis) is chosen as the best measure of success of the various radiotherapeutic techniques utilized in the treatment of patients with corpus cancer limited to the uterus in this series. There were 3 patients with persistent pelvic disease after radiotherapy and 26 central or pelvic side wall recurrences $(9$ cases the site of recurrence is not known). Successful control of local disease carried a $70 \% 5$ year survival probability, while method failure predicted a dismal $19 \%$ survival $(P=0.001)$.

Using the local failure assessment, there was no advantage of statistical significance among the general radiotherapeutic methods utilized, although the approach of external beam alone gave the poorest results (Table 6). The use of 6$8 \mathrm{MeV}$ LINAC photons (in 16 patients) did not improve local failure rates when contrasted to "Orthovoltage" (15 patients) or cobalt-60 therapy ( 26 patients).

Heyman's packing technique was utilized in 55 of the 111 intracavitary applications in the treatment of 103 patients with disease limited to the uterus, while 56 patients had "line sources" in the uterus. Although local disease was more often successfully controlled with the Heyman's approach, the difference only approached statistical significance $(P=0.08)$. Vaginal sources were used in only 66 of the 111 applications. Sixty-two of these sixty-six were via ovoids or colpostats, and the remainder were "line sources" in the vagina. There was no difference in rates of local disease control with either approach.

Recreation of precise radiation dosimetry was not possible because sufficient technical simulation data was not always available. Determination of dose/response relationships or threshold doses for local control was also impossible because of the variation and expression of the intracavitary exposures (recorded either in $\mathrm{rad}$ to Point $\mathrm{A}$ or in milligram/hour).

\section{Outcome of Patients with Prior Radiation Therapy}

A special note is made of the six patients treated with radiation therapy for adenocarcinoma of the endometrium who had had prior pelvic irradiation. Details

TABLE 6

Correlation of Radiotherapeutic Methods with Method Outcome $a$ in the 103 Cases of Stage I AND II UTERINE ADENOCARCINOMA

\begin{tabular}{lcccc}
\hline & \multicolumn{3}{c}{ Radiotherapeutic methods } \\
\cline { 2 - 5 } Method outcome & Intracaviatry & $\begin{array}{l}\text { Intracavitary } \\
\text { and external }\end{array}$ & External & Total \\
\hline Success & 29 & 31 & 5 & 65 \\
Failure & 12 & 13 & 4 & 29 \\
Total & 42 & 44 & 9 & 94 \\
\hline
\end{tabular}

"Method failure is defined as "locally persistent or locally recurrent disease" (nine cases, local status not known). 
of their original radiation were not available. There were four patients with Stage I disease, one patient with Stage II disease, and one with Stage IV disease. There was only one tumor-free survivor at 5 years. Three cases were managed with intracavitary alone $(5000 \mathrm{mg} / \mathrm{hr})$ and one of these died of recurrent pelvic disease at 20 months while the remaining two died of medical causes, tumor free, at 2 and $60+$ months. External beam alone was used in two patients and both had persistent pelvic disease and subsequently died of malignancy at 8 and 16 months. The final patient was treated with 5000 rad whole pelvic external beam and an intracavitary application of $3000 \mathrm{rad}$ to point $\mathrm{A}$, and she is alive, disease free, at 22 months. No major radiation morbidity was noted in any patients within the observation periods.

\section{Management of Radio Persistent or Radio Recurrent Disease}

There were three patients with persistent disease and 46 patients with recurrent metastatic disease (any site) among the 103 cases of Stage I and II corpus cancer. Eighteen of these patients received no further therapy and all died of progressive malignancy by 12 months after the recurrence was detected. Thirty-one patients with recurrence received some form of additional therapy but $58 \%$ were dead of disease by 2 years and only $15 \%$ were predicted to be alive at 5 years. Progestational therapy alone was the most common treatment for recurrence and was used in seven patients. Four patients were managed with progestational agents and cytotoxic drugs; three patients received progestational agents and "palliative" radiation therapy (to extrapelvic metastases). Four patients were treated solely with "palliative" radiation therapy (to extrapelvic sites). Finally, two cases of recurrence were managed with cytotoxic drugs only and two patients with persistent or recurrent local disease had subsequent surgery. In nine cases the management of recurrence was not specifically known. Overall, 43 of the 49 cases of persistent or recurrent disease died of progressive malignancy and only three died of other causes, disease free.

\section{Complications of Therapy}

A massive pulmonary embolus during a radium application accounted for the only treatment-related mortality in this series of 117 patients. Two patients required hospitalization for medical management of radiation proctitis/enteritis but did not require surgery. Both patients had received their external-beam therapy via cobalt unit and both were long-term survivors, free of disease. Two additional patients developed radiation bowel injury subsequently requiring surgery. One patient developed a rectal stricture after treatment with a $250 \mathrm{kV}$ unit and intracavitary radium. She underwent colostomy diversion and died of myocardial infarction 11 months post-treatment. A second patient, also treated with $250 \mathrm{kV}$ external unit in addition to intracavitary radium, developed a complex enterovaginal/ rectovaginal fistula, and underwent successful surgical management and survived 20 years.

There were also a number of other complications that occurred during therapy which subsequently influenced the choice and execution of therapy. One patient developed staphylococcal pneumonia during external-beam therapy and was too 
ill for an intracavitary treatment. Two cases of pyometria required drainage and antibiotics, but intracavitary radium packings were then performed without incident. Endometritis during an intracavitary radium application occurred in one case, and again after successful medical management repacking was accomplished to complete treatment. Finally, two patients had uterine perforation at the time of attempted intracavitary radium application. In both cases, packing was not reattempted. Of the six cases of complications during therapy, four subsequently died of their uterine malignancy.

\section{DISCUSSION}

The fundamental principle of management of adenocarcinoma of the endometrium is to remove the uterus, tubes, and ovaries. Numerous reports [7-9] predict an 89-91\% 5-year survival for Stage I disease managed by surgery. Although recent reviews show a $65-78 \%$ survival for patients managed with radiation therapy alone [2-4], clearly this represents a less desirable approach for primary treatment. It is not the intent of the authors to advocate radiation as the "standard best management" but to reaffirm and clarify its invaluable place as an alternative treatment for selected patients with contraindications to surgery. Indeed, at this time, radiotherapy is the only viable alternative. Chemotherapy or hormonal manipulation produce only rare sustained "complete responses" although trials have been limited to recurrent or advanced disease [10].

Significant advances in all areas of general medicine have decreased the number of "medically inoperable" cases, and the assessment of medical risk can only be made on individual basis by the attending physician. The mean predicted survival for a woman age 70 is about 170 months [11]. Although no standard selection criterion was defined, patients in this series having apparent successful management of their corpus cancer only survived a mean of 71.2 months, demonstrating the impact of their complicating medical conditions.

Prior or concomitant malignancy should also be considered in selection of therapy for uterine cancer. The high incidence $(24 \%)$ of other malignancies is not surprising in this age group, but it is significant that tumor deaths in patients with second primaries were most often due to the nonuterine cancer $(10 / 15)$. Performance status is also a valuable criterion in selection of patients for primary radiation therapy. It reflects the overall health of the patient and the impact of medical illnesses on the ability of the patient to function normally. Radiotherapy should cause the least deterioration of a compromised performance status.

This series represents a unique group of patients with endometrial cancer. Only $52 \%$ of the cases were Stage I; only $64 \%$ of the cases had "endometrioid" type of adenocarcinoma; only $44 \%$ of the cases had Grade I lesions. In this context, 49.6\% 5-year predicted actuarial survival for the 117 patients is not surprising.

Because of the impact of associated medical conditions and because of the inability to control extrapelvic disease even with "standard surgical management," the focus of therapy should be effective local pelvic control. The overall local failure rate (for disease limited to the uterus) was $30.8 \%$ (29/94). The available data for analysis of these 94 patients would indicate that the addition of external- 
beam therapy to the intracavitary approach did not significantly improve local control or increase survival. Although several reports [2,12] support the use of external beam as part of a primary radiation schema for corpus cancer, others $[4,13]$ consider the radium packing the most important component.

External-beam therapy is designed to increase the pelvic side wall (i.e., pelvic nodal) doses. This is done, however, at the expense of the central intracavitary radium dose. "Sterilization" of the uterine cavity of tumor has been reported in 59-78\% of the cases reviewed by Strickland [13] and Joelsson et al. [14] when preoperative intracavitary radiation was used. This is contrasted to only a $38.9 \%$ sterilization rate when preoperative external-beam radiation was used [15]. Compromise of the intracavitary packing dose therefore may decrease uterine control with primary radiation therapy.

The packing technique itself does deliver radiation to the side wall or pelvic nodal areas and this can be as high as 3000-4000 rad as demonstrated by Landgren et al. [4] Moreover, although recent emphasis has been placed on nodal disease associated with adenocarcinoma of the endometrium, estimates of pelvic nodal metastases in Stage I and II cases (all grades and histologic types) are a relatively low 10\% [16]. Clearly the main treatment emphasis for the majority of patients with disease confined to the uterus should be the uterus itself.

Since, in this series, the addition of external irradiation did not improve local failure rates or survival, it follows that no significant advantage was shown when megavoltage external radiation units were used to deliver the external-beam therapy. This agrees with the observations of Wilson et al. [15].

The Heyman packing technique, with large endometrial dosages, appeared to give better results than the use of "line sources" in the uterus, but the advantages were of borderline statistical significance in this series. The Heyman approach requires more care and skill to avoid complications [17]. Another problem with the Heyman technique is that dosimetry curves can only be approximated because of the unknown "filtering" that occurs due to the capsules themselves [18].

Recurrent disease, in this series, was almost universally fatal, and no specific form of management emerged as superior. It is felt that the general compromised medical condition of the patients reviewed was not conducive to aggressive chemotherapy which may explain in part the dismal outcome, but agents with high sustained activity against endometrial carcinoma are still not available [19]. The "cost" of radiotherapy was a treatment mortality rate of $0.8 \%$, an overall complication rate of $6.8 \%$, and a $3.4 \%$ incidence of major complications (i.e., those requiring hospitalization or surgery at some period after treatment). The impression is that the use of "megavoltage" external therapy led to fewer significant complications.

\section{SUMMARY}

The present series of 117 patients with adenocarcinoma of the endometrium treated by radiation therapy alone continues to confirm that surgery, whenever possible, gives superior results. In the selected high-risk patient, however, results of primary irradiation are quite acceptable especially in view of minimal treatmentrelated morbidity and mortality. Selection of patients for primary radiotherapeutic 
treatment must be individualized. The cornerstone of successful radiation treatment remains intracavitary radiation. The Heyman packing technique may offer an advantage in local control, but the technique is more difficult and not universally applicable. Until more active hormonal or chemotherapeutic agents evolve, radiation therapy is the only alternative for the management of adenocarcinoma of the endometrium in patients with contraindications to surgery.

\section{REFERENCES}

1. Heyman, J., Reuterwall, O., and Benner, S. The radiumhemmet experience with radiotherapy in cancer of the corpus of the uterus, Acta Radiol. 22, 114 (1941).

2. Abayomi, O., Tak, W., Emami, B., and Anderson, B. Treatment of endometrial carcinoma with radiation therapy alone, Cancer 49, 2466 (1982).

3. Fayos, J. V., and Morales, P. H. Carcinoma of the endometrium results of treatment, Radiat. Uncol. Biol. Phys. 6, 571 (1980).

4. Landgren, R. D., Fletcher, G. H., Gallagher, H. S., Declos, L., and Wharton, J. T. Treatment failure sites according to irradiation technique and histology in patients with endometrial cancer, Cancer 40, 131 (1977).

5. Joslin, C. A. F., and Peel, K. R. The uterus, in Treatment of cancer (K. E. Halnan, Ed.), Igaku-Shoin, New York (1982).

6. Hendrickson, M., Ross, J., Eifel, P. J., Cox, R. S., Martinez, A., and Kempson, R. Adenocarcinoma of the endometrium: Analysis of 256 cases with carcinoma limited to the uterine corpus, Gynecol. Oncol. 13, 373 (1982).

7. Underwood, P. B., Jr., Lutz, M. H., Kreutner, A., Miller, M. C., III, and Johnson, R. D. Jr. Carcinoma of the endometrium: Radiation followed immediately by operation Amer. J. Obstet. Gynecol. 128, 86 (1977).

8. Alders, J., Abeler, V., and Kolstad, P. Postoperative external irradiation and prognostic parameters in Stage I endometrial carcinoma, Obstet. Gynecol. 56, 419 (1980).

9. Malkasian, G. D. Carcinoma of the endometrium. Effect of stage and grade on survival, Cancer 41, 996 (1978).

10. Willson, J. K. V., Ozols, R. F., Lewis, B. J., and Young, R. C. Current status of therapeutic modalities for treatment of gynecologic malignancy with emphasis on chemotherapy, Amer. J. Obstet. Gynecol. 141, 81 (1981).

11. Facts of life and death, DHEW 79/1222, U.S. Dept. HEW (1978).

12. Cox, J. D., Ritsuko, K., Wilson, J. F., and Greenberg, N. Locally advanced adenocarcinoma of the endometrium: Results of radiation with and without subsequent hysterectomy, Cancer 45, 715 (1980).

13. Strickland, P. Carcinoma corporis uteri: A radical intracavitary treatment, Clin. Radiol. 16, 112 (1965).

14. Joelsson, I., Sandri, A., and Kottmeier, H. L. Cancer of the uterine corpus, Acta Radiol. Suppl. 334, 3 (1973).

15. Wilson, J. F., Cox, J. D., Chahbazian, C. M., del Regato, J. A. Time dose relationships and endometrial adenocarcinoma: Implications of the interval from external beam irradiation to surgery, Radiat. Oncol. Biol. Phys. 6, 597 (1980).

16. Disaia, P. J., and Creasman, W. T. Clinical gynecologic oncology, Mosby, St. Louis (1981).

17. Landgren, R. C., Fletcher, G. H., Declos, L., and Wharton, J. T. Irradiation of endometrial cancer in patients with medical contraindications to surgery or with unresectable lesions, Amer. J. Roentgenol. Rad. Ther. Nucl. Med. 126, 141 (1976).

18. Wollin, M., Kagan, A. R., and Kwan, D. K. Radiation dose calculations in endometrial cancer treated with Heyman's capsules or tandem, Gynecal. Oncol. 13, 37 (1982).

19. Horton, J., Elson, P., Gordon, P., Hahn, R., and Creech, R. Combination chemotherapy for advanced endometrial cancer, Cancer 49, 2441 (1982). 